\title{
Uma História da
}

\section{Habitabilidade Cósmica}

\author{
Amedeo Balbi \\ Departamento de Física Universidade de Roma Tor Vergata \\ Traduzido por Daniel de Pinho Barreiros
}

Correspondência | balbi@romaz.infn.it

Citation | Balbi, Amedeo. 2020. “Uma História da Habitabilidade Cósmica.” Traduzido por Daniel de Pinho Barreiros. Journal of Big History IV (2): 54-59.

DOI | https://doi.org/10.22339/jbh.v4i2.4222

Resumo Nossa compreensão do universo é baseada no modelo cosmológico do Big Bang, que descreve um universo em expansão cujo desenvolvimento começou 13,8 bilhões de anos atrás, a partir de um estado quente e denso. Este modelo introduz uma forte perspectiva evolutiva e histórica na descrição de muitos fenômenos físicos observados, incluindo a origem da vida e sua possível distribuição no universo. Discuto como é relevante para a astrobiologia e para a SETI (a busca de inteligência extraterrestre) levarmos em conta, apropriadamente, o "quadro geral" e seu desdobramento temporal.

\section{Introdução}

No século passado, aprendemos muito sobre a história do universo. Uma das coisas mais importantes que aprendemos é precisamente que o universo tem uma história. Isso significa que sabemos que o universo observável nem sempre foi do jeito em que se encontra hoje. Ele tem evoluído por 13,8 bilhões de anos, e mudou

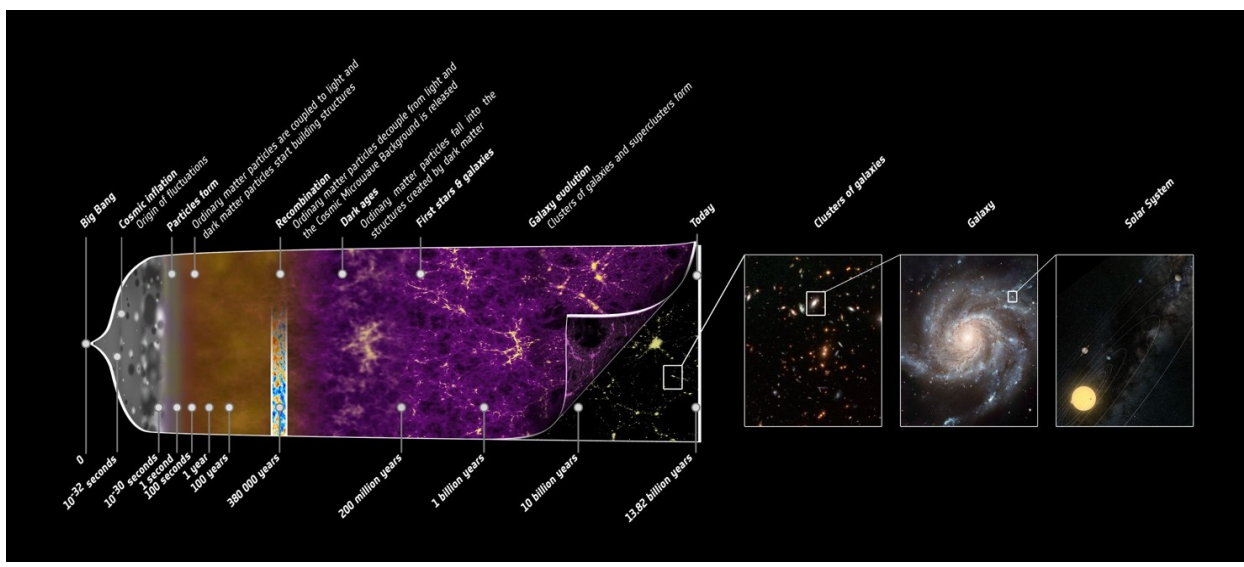

Figura 1: Il "quadro generale" della storia dell'universo secondo il modello cosmologico standard del big bang (Credito immagine: NASA) drasticamente durante esse período. Tudo começou no passado a partir de um estado muito simples e indiferenciado, muito próximo do equilíbrio (pelo menos em certo sentido, a ser especificado posteriormente) e depois, com o passar do tempo, complexidade e estrutura emergiram lentamente (Figura 1). A partir de um plasma primordial quente, composto de partículas livres, as forças da natureza formaram galáxias, estrelas, planetas e moléculas. As principais perguntas para a astrobiologia são as seguintes: Como a vida se encaixa no esquema geral do universo? E quão comum é isso? A vida na Terra é apenas um evento casual, sem equivalente no resto do universo, ou é parte de um fenômeno genérico que aconteceu por muitas vezes em outros lugares? O ponto principal deste artigo é enfatizar como a perspectiva da Grande História é essencial para obter uma boa compreensão de tais questões.

Para um físico ou um cosmologista, a maioria das coisas que aconteceram na história do universo são de previsão bastante simples. Por exemplo, sabendo que no começo do universo havia uma dada amplitude em termos de flutuações de densidade no plasma primordial, seria bastante fácil prever 
que, depois de algum tempo, estrelas e galáxias surgiriam. Do ponto de vista da física, o fato de o universo estar cheio de estrelas não seria uma surpresa, mesmo para alguém que nunca viu uma estrela. Contudo, quando tentamos incluir a vida num enquadramento cósmico, as coisas são muito mais complicadas. No momento não há como prever, a partir dos princípios primordiais, por exemplo, a época em que a vida apareceu pela primeira vez, ou sua frequência e distribuição no universo.

De fato, em um cenário em que o universo existiu e evoluiu por 13,8 bilhões de anos-ou seja, no modelo do "Big Bang quente" que constitui nossa melhor descrição atual da história cósmica-até a existência de uma única instância de vida em um único planeta apresenta - se como um enigma. Para entender o porquê, compare a situação com a prevista pelo agora desacreditado modelo de estado estacionário (Bondi e Gold 1948), que postulava um universo que existe para sempre no mesmo estado em que o observamos hoje: nesse universo, havia tempo suficiente (de fato, uma quantidade infinita de tempo) para produzir, algures, até a mais improvável combinação de moléculas. Portanto, em um universo estacionário e eterno, não deveríamos nos surpreender ao descobrir que a vida existe. Por outro lado, a mera existência de um único exemplo de vida em um universo com uma idade finita-o tipo de universo em que realmente vivemos-pede uma explicação. E de igual importância é o seguinte: a avaliação da probabilidade do surgimento da vida não pode ser facilmente desvinculada da evolução geral do cosmos e de suas características específicas, tais como os valores dos parâmetros físicos e das constantes que governam seu comportamento (Barrow 1998; Rees 1972).

História Cósmica, Complexidade e Vida

No modelo do Big Bang, o surgimento da vida-ou, pelo menos, o surgimento das condições para a emergência da vida-requer uma série de etapas preliminares. Primeiro, o universo precisa criar os núcleos de elementos leves no plasma primordial; então ele precisa formar estrelas, e as estrelas precisam fundir elementos mais pesados em seus núcleos, até que sua abundância seja grande o suficiente para formar planetas rochosos em torno das estrelas da sequência principal. Então, nuvens moleculares no espaço interestelar precisam formar moléculas orgânicas, que podem acabar alcançando atmosferas e superfícies planetárias, enquanto colisões e impactos com corpos gelados precisam transportar água suficiente para planetas na zona habitável de suas estrelas-onde temperaturas e pressão atmosférica são compatível com a presença de lagos e oceanos. Somente após todas essas etapas, é possível que moléculas replicadoras apareçam-e talvez o metabolismo, os micro-

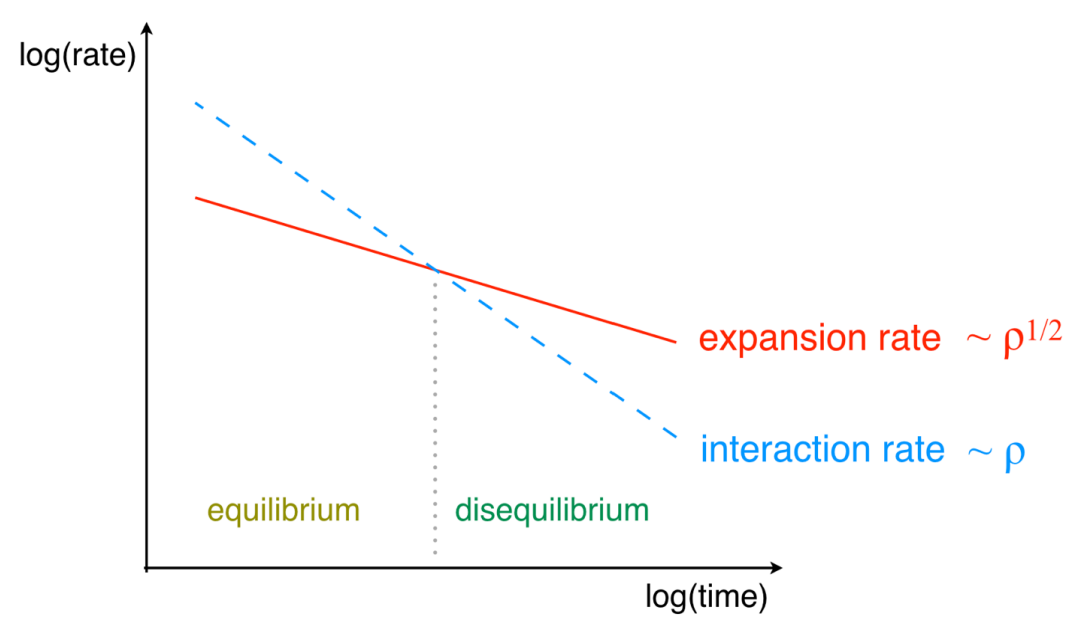

Figura 2: Il tasso di espansione dell'universo e il tasso di interazione di qualsiasi processo a due corpi dipendono in modi diversi dalla densità media dell'universo (che, a sua volta, diminuisce come una potenza del tempo cosmico). Ciò implica che ogni interazione alla fine va fuori equilibrio, poiché la sua velocità diventa più piccola della velocità di espansione dell'universo. organismos, a vida complexa e a inteligência.

Isso é verdade, independentemente da abundância de vida no universo-sendo ela generalizada ou existente em apenas alguns locais em todo o universo observável. O problema da origem da vida não pode ser resolvido sem uma compreensão desse quadro geral. Este é o contexto cósmico geral no qual todos os passos necessários para o aparecimento da vida ocorreram. É importante notar que todos esses passos podem ser organizados em uma ampla narrativa evolutiva (Chaisson 2001), e isso, por sua vez, torna a perspectiva histórica crucial para interpretar o quadro físico.

O próprio fato de haver uma tendência evolutiva pode parecer contra-intuitivo a princípio. A tendência óbvia de simplicidade para complexidade que observamos no universo parece contrariar nossa compreensão ingênua da termodinâmica: sabemos que a simplicidade é mais provável que a complexidade e que a desordem vem depois da ordem, e não vice-versa. $\mathrm{Na}$ verdade, no entanto, não há nada de estranho nisso. De fato, a aparência espontânea de ordem e 
auto-organização no universo é possível por duas razões, ambas diretamente relacionadas ao comportamento cosmológico geral. Primeiro, em um universo em expansão, qualquer processo tende a sair de equilíbrio em algum momento (Figura 2). Isso se deve ao fato de que o tempo médio necessário para que, eventualmente, qualquer interação ocorra se torna maior do que a taxa na qual o universo está se expandindo (ou seja, o tempo que leva para o universo dobrar seu tamanho), porque, embora essas duas taxas dependam da densidade geral do universo, elas dependem dessa densidade de maneiras diferentes (Balbi 2018). Quando isso acontece, a interação não pode ser rápida o suficiente para manter o equilíbrio. Assim, o desequilíbrio pode surgir de um estado inicial muito uniforme.

A segunda razão está relacionada ao papel termodinâmico da gravidade. Quando pensamos em entropia, a imagem padrão dos livros didáticos é a de um gás em uma caixa (Figura 3). Se o gás começa com todas as moléculas agrupadas em um canto da caixa, esperamos que mais tarde assuma um estado mais simples, mais uniforme e desordenado, onde as moléculas estejam homogeneamente espalhadas por toda a caixa. Portanto, tendemos a associar alta entropia com alta uniformidade. Mas, se incluirmos a gravidade nas imagens, as coisas seguem o caminho oposto: a aglomeração e a entropia aumentam na mesma direção. De fato, apesar da aparência lisa, o estado inicial do universo está muito longe da mais intensa desordem possível. $\mathrm{Na}$ linguagem termodinâmica, um universo em expansão tem muitas maneiras de aumentar sua entropia e, ao mesmo tempo, produzir estruturas complexas

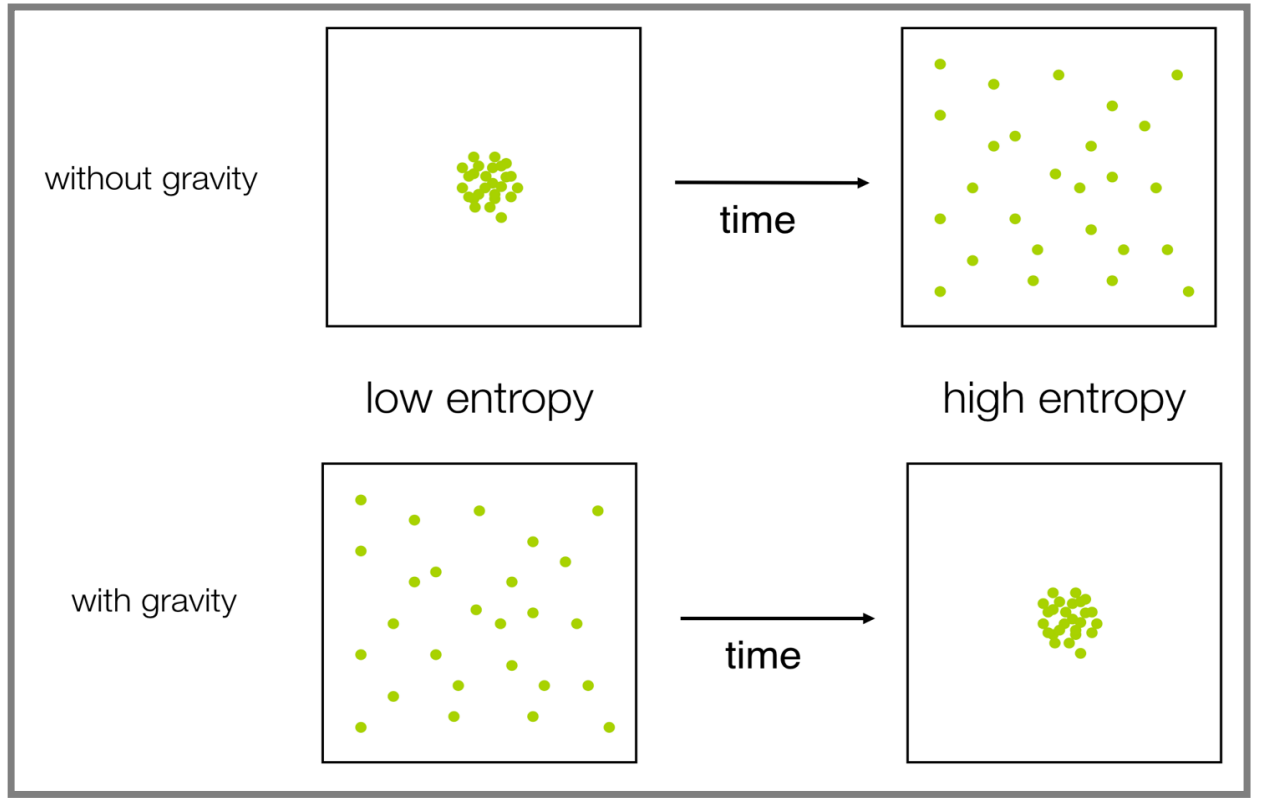

Figura 3: In presenza di gravità, la crescita dell'entropia non corrisponde a una maggiore uniformità, come nell'immagine standard del "gas in una scatola" (pannello superiore): piuttosto, essa porta a una maggiore disuniformità (pannello inferiore). Questo è cruciale per spiegare l'emergere spontaneo, e in qualche modo controintuitivo, della struttura nell'universo col passare del tempo.

transitórias (ver, por exemplo, Carroll 2010).

Tudo isso, claro, é crucial para a possibilidade de vida. A vida prospera no desequilíbrio produzido pela evolução do universo. Pense, por exemplo, na luz do sol atingindo a superfície da Terra. Os fótons vindos do Sol têm baixa entropia em comparação com os que deixam a Terra em direção ao espaço. Em meio a esse fluxo de fótons partindo de uma fonte quente de baixa entropia (o Sol) em direção a um dissipador frio de alta entropia (o vácuo espacial), existe a Terra, e a vida nela. A vida é possível porque há trabalho a ser extraído deste motor termodinâmico operando entre diferentes temperaturas (Lineweaver e Egan 2008). Vários estudos começaram a adotar um ponto de vista explicitamente termodinâmico ao considerar a vida no contexto

cósmico (ver, por exemplo, Frank, Kleidon e Alberti 2017). Uma maneira de enquadrar a questão é a de ver a vida como uma maneira eficiente (ou mais eficiente que os sistemas não-vivos) de usar energia livre e produzir entropia, de acordo com o princípio da máxima entropia (MEP, ver, por exemplo, Kleidon 2010a, 2010b). .

Vemos então que há um forte aspecto histórico (no sentido de uma relação profunda com o fluxo do tempo) quando tentamos entender o lugar da vida no universo. Isto não é apenas verdade no que diz respeito à história cósmica passada, mas também ao considerar sua evolução futura. Não temos certeza do que vai acontecer (de fato, podemos nunca ter certeza, ver Krauss e Turner, 1999), mas se o universo continuar se expandindo a uma taxa acelerada (como sugerem as observações atuais), eventualmente se tornará frio e sem estrutura, aproximando-se do equilíbrio termodinâmico (Adams e Laughlin 1997). Haverá uma eternidade em que basicamente nada de complexo poderá acontecer, já que o universo chegará à morte térmica, a mais alta entropia possível. Isso coloca o tempo presente em uma posição ainda mais estranha. 
Estamos vivendo uma época fugaz na história geral do universo - um pequeno intervalo temporário em que pode haver estrelas, planetas, moléculas e vida, entre dois éons igualmente descaracterizados. Uma conseqüência potencialmente profunda disso é que, embora o copernicanismo seja um bom princípio orientador quando aplicado ao espaço, ele certamente está errado quando aplicado ao tempo. Nossa época é bastante atípica, e devemos levar isso em consideração ao prever a frequência e distribuição da vida no universo, ou ao julgar sua probabilidade (Cirkovic e Balbi 2019).

\section{Quando o Universo Se Tornou Habitável?}

Levar em consideração a variável temporal é de extrema importância para a astrobiologia e para a SETI. Um aspecto do papel do tempo é o de considerar "quando" o universo se torna adequado para a vida. Isso pode parecer uma pergunta óbvia (especialmente na perspectiva da Grande História), mas surpreendentemente recebeu pouca atenção até pouco tempo atrás. De fato, geralmente pensamos na habitabilidade apenas como uma questão de "onde" podem existir as condições necessárias para a origem e a manutenção da vida (ver, por exemplo, Cockell et al 2016). Por exemplo, há muito interesse em encontrar planetas em torno da zona habitável de outras estrelas-ou seja, onde a temperatura pode ser compatível com a presença estável de água líquida na superfície (Kopparapu et al 2013). Também foi feito algum esforço para a identificação de locais adequados em nossa galáxia, onde os planetas habitáveis, e talvez a vida, sejam mais prováveis-ou seja, uma “zona habitável galáctica” (Lineweaver 2001; Lineweaver et al 2004; Gonzales 2005). Mas comparativamente pouco esforço foi dedicado a avaliar em que época da história cósmica a vida passou a ser possível (Dayall et al 2016; Loeb et al 2016). Está claro na discussão

anterior que as condições corretas não estavam presentes em nosso universo desde o início, e podem não estar presentes em um futuro distante.

Existem exemplos conhecidos de processos astrofísicos com uma forte dependência temporal que podem potencialmente afetar a habitabilidade, não apenas na escala local dos sistemas planetários, mas até mesmo em escala galáctica. Por exemplo, sabemos que durante o início da história de nossa galáxia (cerca de 8 bilhões de anos atrás), o buraco negro supermassivo em seu centro estava ativo-ou seja, estava acumulando matéria a uma taxa muito alta. Como conseqüência disso, altos níveis de radiação ionizante foram emitidos a partir do núcleo galáctico, e isso pode ter tido um impacto na habitabilidade geral da galáxia.

Exoplanetas rochosos próximos o suficiente do buraco negro (dentro de mil anos-luz, ou seja, na região

protuberante da galáxia) enquanto esteve ativo (período esse que pode ter durado por aproximadamente 100 milhões de anos), podem ter perdido uma fração substancial de suas atmosferas (Balbi e Tombesi 2017). Além disso, a vida nesses planetas pode ter sofrido sérios danos biológicos.

É claro que este é um tópico muito amplo e complexo, e estamos apenas começando a arranhar sua superfície: é difícil dizer, por exemplo, se a radiação ionizante é prejudicial à vida ou, de alguma forma, também necessária, talvez para induzir mutações e outros efeitos biogênicos (Lingam et al 2019). Claramente, há muito o que aprender ao estudar a conexão entre a história do universo e sua habitabilidade, tanto em pequenas como em grandes escalas. Construir uma história de habitabilidade cósmica seria um subcampo potencialmente fecundo da Grande História.

\section{Os Aspectos Temporais do SETI}

Compreender a história da

habitabilidade cósmica também teria um forte impacto na busca por vida inteligente em outras partes do universo. Infelizmente, o fator temporal foi quase totalmente ignorado nos estudos da SETI (Cirkovic 2004). Um campo em que o tempo entra em jogo é o das propriedades causais necessárias a qualquer sinal de vida que possamos detectar. Em outras palavras, quando procuramos civilizações capazes de se comunicar conosco e que eventualmente habitem nossa galáxia, temos de atentar para o fato de que existem requerimentos estritos a serem cumpridos por um sinal

eletromagnético (ou, de forma mais geral, qualquer interação física) para que ele seja captado por nós, hoje. A título de ênfase, pode-se pensar em uma mensagem de rádio emitida a partir de um local a mil anos-luz do Sol: se essa comunicação cessou antes de mil anos atrás, não podemos mais observá-la. Isso pode parecer a princípio um fato trivial, mas apresenta implicações profundas (Balbi 2017; Grimaldi 2017). Por exemplo, isso implica que qualquer civilização tecnológica da qual possamos encontrar evidências empíricas deve ser ou muito longeva, ou aproximadamente contemporânea a nós. Isso também implica que as chances de descobrir vida inteligente fora da Terra dependem de como a vida foi distribuída no tempo ao longo da história cósmica. Se somos retardatários e a maioria das outras civilizações já foi extinta, podemos estar sozinhos. Por outro lado, se a vida começou a aparecer apenas muito recentemente, e o universo está apenas 
acordando agora, podemos ser uma das primeiras civilizações tecnológicas.

Isso pode lançar uma nova luz sobre antigos enigmas, como a incompatibilidade entre o grande número de locais potencialmente habitáveis no universo e a ausência de evidências de vida inteligente além da Terra (geralmente resumido pela pergunta de Fermi: "Onde estão todos?”). Se o universo não era tão favorável à vida no passado como é hoje, então as perguntas mudam e o quebra-cabeça assume novos aspectos. Avaliar as chances de sucesso da SETI, ou mesmo interpretar resultados negativos, é algo que precisa considerar conhecimentos acerca de como a propensão à vida em nosso universo mudou ao longo do tempo, perspectiva essa que é inseparável dos aspectos históricos e evolutivos do cenário cosmológico.

\section{Conclusão}

$\mathrm{O}$ fato de vivermos em um universo em expansão com uma idade finita, cujo estado médio mudou drasticamente ao longo do tempo, introduz uma perspectiva histórica em muitos dos processos físicos que a ciência tenta explicar. Argumentei aqui que o problema da origem da vida, na Terra e em outros lugares, deve abraçar completamente esse ponto de vista histórico e evolutivo. Investigar a história da habitabilidade cósmica-isto é, da propensão do universo a hospedar a vida-pode iluminar muitos aspectos da pesquisa contemporânea em astrobiologia e SETI. Eventualmente, essa é mais uma indicação de que a adoção de uma perspectiva fundada na Grande História pode ter consequências profundas para a compreensão de nosso lugar no cosmos.

\section{Referências}

Adams, Fred C., and Gregory Laughlin. 1997. “A Dying Universe: The Long Term Fate and Evolution of Astrophysical Objects." Reviews of Modern Physics 69 (2): 337, doi:10.1103/ RevModPhys.69.337.

Balbi, Amedeo. 2013. "Cosmology and Time." EPJ Web of Conferences 58, doi:10.1051/epjconf/20135802004.

Balbi, Amedeo. 2018. "The Impact of the Temporal Distribution of Communicating Civilizations on Their Detectability." Astrobiology 18 (1): 54 .

Balbi, Amedeo, and Francesco Tombesi. 2017. "The Habitability of the Milky Way during the Active Phase of Its Central Supermassive Black Hole.” Scientific Reports 7, doi:org/10/1038/s41598-017-16110-o.

Barrow, John D. 2008. Cosmology and the Origin of Life. November 30, 1998. Invited presentation. Varenna Conference on the Origin of Intelligent Life in the Universe.

ArXiv:astro-ph/9811461.

Bondi, H., and T. Gold. 1948. "The Steady-state Theory of the Expanding Universe." Monthly Notices of the Royal Astronomical Society 108 (3): 252-270, doi.org/10.1093/ mnras/108.3.252.

Carroll, Sean. 2010. From Eternity to Here: The Quest for the Ultimate Theory of Time. New York: Dutton.

Chaisson, Eric J. 2001, Cosmic Evolution: the Rise of Complexity in Nature. Cambridge, MA: Harvard University Press.

Ćirković, Milan M. 2004. "The Temporal Aspect of the Drake Equation and SETI." Astrobiology 4 (2): 225.
Ćirković, Milan M., and Amedeo Balbi. 2019. "Copernicanism and the Typicality in Time." International Journal of Astrobiology 19 (2): 1-9, doi.org/10.1017/S1473550419000223.

Cockell, C. S., T. Bush, C. Bryce, S. Direito, M. Fox-Powell, J. P. Harrison, H. Lammer, et al. 2016. "Habitability: a Review." Astrobiology 16 (1): 89-117, doi.org/10.1089/ ast.2015.1295.

Dayal, Pratika, Martin Ward, and Charles Cockell. 2016. "The Habitability of the Universe through 13 Billion Years of Cosmic Time." ArXiv:16o6.09224.

Frank, Adam, Axel Kleidon, and Marina Alberti. 2017. "Earth as a Hybrid Planet: The Anthropocene in an Evolutionary Astrobiological Context." Anthropocene 19: 13.

Gonzalez, Guillermo. 2005. "Habitable Zones in the Universe." Origin of Life and Evolution of the Biospheres 35 (6): 555-606.

Grimaldi, Claudio. 2017. "Signal Coverage Approach to the Detection Probability of Hypothetical Extraterrestrial Emitters in the Milky Way." Scientific Reports 7 (46273).

Kleidon, A. 2010. "A Basic Introduction to the Thermodynamics of the Earth System Far from Equilibrium and Maximum Entropy Production." Philosophical Transactions of the Royal Society of London. Series B, Biological Sciences. 365 (1545): 1303.

Kleidon, Axel. 2010. "Life, Hierarchy, and the Thermodynamic Machinery of Planet Earth. Physics of Life Reviews 7 (4): 424.

Kopparapu, Ravi Kumar, Ramses Ramirez, James F. Kasting, Vincent Eymet, Tyler D. Robinson, Suvrath 
Mahadevan, Ryan C. Terrien, et al. 2013. "Habitable Zones around Main-sequence Stars: New Estimates." Astrophysical Journal, 765 (131): 1-16.

Krauss, Lawrence M., and Michael S.

Turner. 1999. "Geometry and Destiny." General Relativity and Gravitation 31: 1453.

Lineweaver, C. H. 2001. "An Estimate of the Age Distribution of Terrestrial Planets in the Universe: Quantifying Metallicity as a Selection Effect." Icarus 151: 307-313.

Lineweaver, C. H., Y. Fenner, and B.

K. Gibson. 2004. "The Galactic Habitable Zone and the Age Distribution of Complex Life in the Milky Way." Science 303 (5654): 59-62.

Lineweaver, Charles H. 2005. Cosmological and Biological Reproducibility: Limits of the Maximum Entropy Production Principle. In Kleidon, A., and R. Lorenz. Nonequilibrium Thermodynamics and the Production of Entropy: Life, Earth, and Beyond 2: 67.

Lineweaver, Charles H., and Chas A. Egan. 20o8. Life, Gravity and the Second Law of Thermodynamics. Physics of Life Reviews 5(4): 225.

Lingam, Manasvi, Idan Ginsburg, and Shmuel Bialy. 2019. "Active Galactic Nuclei: Boon or Bane for Biota?" Astrophysical Journal 877: 62.

Loeb, Abraham, Rafael A. Batista, and David Sloan. 2016. "Relative Likelihood for Life as a Function of Cosmic Time." Journal of Cosmology and Astroparticle Physics 8: 10.

Rees, M. J. 1972. “Cosmological Significance of e2/Gm2 and Related Large Numbers." Comments on Astrophysics and Space Physics 4: 179185. 